\title{
Evaluation of the Ohmeda 3700 pulse oximeter
}

\author{
A R H WARLEY, J H MITCHELL, J R STRADLING \\ From the Chest Clinic, Churchill Hospital, Oxford
}

ABSTRACT Arterial oxygen saturation values $\left(\mathrm{SaO}_{2}\right)$ from $60 \%$ to $98 \%$ were measured by the Ohmeda 3700 pulse oximeter with the three types of probe available and compared with values of oxygen saturation estimated from direct arterial sampling (arterial oxygen and carbon dioxide $\vec{\infty}$ tensions and $\mathrm{pH}$ ) on 65 occasions. The response time of the oximeter was measured after a suddent rise in inspired oxygen concentration. Artefact rejection was assessed by arterial compression prox $ㅇ$ imal to the probe site, and by simultaneous recordings of overnight $\mathrm{SaO}_{2}$ on opposite hands. The ability to recreate patterns of oscillating $\mathrm{SaO}_{2}$ from the data stored in the oximeter was also investigated. With the best probe system the oximeter measured $\mathrm{SaO}_{2}$, relative to arterial values estimated from $\mathrm{PaO}_{2}$, with a mean (SD) difference of $-0.4 \%(1 \cdot 8 \%)$. The response time was com $\frac{3}{6}$ parable with those of previous oximeters. It was not possible to generate artefactual dips in excess of $2 \% \mathrm{SaO}_{2}$, and the dual overnight recordings rarely showed even small dips on one tracing alone $\vec{\bullet}$ The stored data can recreate oscillating $\mathrm{SaO}_{2}$ signals with wavelengths down to about 35 seconds, but not below. The Ohmeda 3700 pulse oximeter appears to be suitable for unattended overnight: recordings of $\mathrm{SaO}_{2}$.

The Ohmeda Biox 3700 pulse oximeter is a new continuous monitor of arterial oxygen saturation and pulse rate. Its easy portability, the availability of finger as well as ear probes, eight hours of internal memory, and apparently better ability to reject low quality signals should constitute important advantages over previous oximeters, particularly for unattended overnight recordings of arterial oxygen saturation $\left(\mathrm{SaO}_{2}\right)$.

We have tested aspects of the machine's performance with particular attention to inaccuracies that might occur during unattended overnight recordings. These include comparison of the oximeter $\mathrm{SaO}_{2}$ with values obtained from arterial sampling, speed of response, artefact rejection, and the ability of the stored data to recreate the original pattern of $\mathrm{SaO}_{2}$ oscillations.

\section{Methods}

STUDY 1: COMPARISON WITH DIRECT ARTERIAL SAMPLING

Sixty five simultaneous comparisons between direct arterial samples taken for $\mathrm{SaO}_{2}$ estimation and all three probes (finger, flex, and ear) connected to three

Address for reprint requests: Dr J Stradling, Osler Chest Unit, Churchill Hospital, Oxford OX3 $7 \mathrm{LJ}$.

Accepted 1 April 1987 oximeters were obtained. Two normal subjects市 breathed nitrogen at varying concentrations through a Venturi mask and eight hypoxic, non-smokingo inpatients breathed various fractional inspired concentrations of oxygen $\left(\mathrm{FiO}_{2}\right)$ to determine the most appropriate concentration of long term supplemental oxygen, providing a range of $\mathrm{SaO}_{2}$ values from $60 \%$ 을 to $98 \%$. The flex probe was held in place over the tip of a finger by purpose made sticky tape supplied by the company. The finger probe is a more bulky, thim-3. ble like device, which slips over the end of the finger, and the ear probe is like a clothes peg, similar to that from the Biox $2 \mathrm{~A}$ oximeter. When steady state condi-o tions had been achieved at a particular $\mathrm{FIO}_{2}$, blood? was withdrawn from an indwelling radial artery can 0 nula inserted at the beginning of the experiment Arterial oxygen tension $\left(\mathrm{PaO}_{2}\right)$ and carbon dioxidễ tension $\left(\mathrm{PaCO}_{2}\right)$ and $\mathrm{pH}$ were measured on a bloodn gas machine (ABL-2, Radiometer; Copenhagen). If any of the three displayed $\mathrm{SaO}_{2}$ values changed by more than $2 \%$ during the period of blood withdrawak or the previous 30 seconds, the sample was not analysed. Haemoglobin saturation was estimated froms blood gas values with a Severinghaus blood gas calcu= lator. ${ }^{5}$

STUDY 2: SPEED OF RESPONSE

The three different probes from the Ohmeda 3700 and? ear probes from the Hewlett-Packard 47201A or Biox 
2A were connected to either ear, finger, or toe as appropriate with the outputs displayed on a chart recorder. After steady state conditions had been achieved with low $\mathrm{FIO}_{2}$, a mask delivering $100 \%$ oxygen was rapidly substituted. The response time was recorded from the first deep breath of $100 \%$ oxygen to the start of the rise in $\mathrm{SaO}_{2}$.

\section{STUDY 3: ASSESSMENT OF ABILITY TO DETECT}

ARTEFACT

The functioning of the oximeter requires pulsatile blood within the light path to differentiate arterial blood from non-pulsatile capillary or venous blood. An inadequate pulse could lead to falsely low $\mathrm{SaO}_{2}$ values, and therefore the warning "low quality signal" is displayed.

(a) In three normal subjects an arterial cuff was applied to the limb proximal to the flex probe, and repeatedly inflated to above systolic blood pressure. As soon as "low quality signal" was indicated on the display panel the fall in $\mathrm{SaO}_{2}$ (if any) was noted; the limb was then moved in an attempt to simulate pulsatile blood flow and thus deceive the instrument.

(b) Two flex probes attached to different oximeters were worn on opposite hands by five healthy subjects overnight. The paired eight hour $\mathrm{SaO}_{2}$ tracings were then inspected visually for differences, particularly $\mathrm{SaO}_{2}$ dips appearing on only one of the tracings, which would be likely to be artefact.

STUDY 4: EFFECT OF THE 12 SECOND SAMPLING ALGORITHM ON SIGNAL SHAPE

The Ohmeda 3700 memory saves $\mathrm{SaO}_{2}$ and pulse rate values every 12 seconds. The actual value of $\mathrm{SaO}_{2}$ stored is the lowest that occurred in the previous 12 seconds, and the pulse rate stored is the value which was synchronous with the $\mathrm{SaO}_{2}$.

The ability of the algorithm to reproduce the original $\mathrm{SaO}_{2}$ signal accurately was assessed by simulating an oscillating $\mathrm{SaO}_{2}$ signal on a $\mathrm{BBC}$ "B" computer and sampling this signal with the algorithm used by the Ohmeda 3700. Simulated cycle lengths of $\mathrm{SaO}_{2}$ from 30 to 70 seconds were used (similar to that seen in sleep apnoea) and a visual comparison was made between the original and the sampled tracings.

\section{Results}

The Ohmeda 3700 proved simple to use. It was usually easy to find a suitable finger on which the flex probe could be sited to give a strong signal, relatively immune from movement artefact. As the signal becomes weaker it becomes more sensitive to artefact because increased amplification is necessary. The measurement of pulse rate is more susceptible to movement artefact than $\mathrm{SaO}_{2}$, although it is accurate when the probe site is not moving. The purpose made tapes to attach the flex probe to a finger are white and sometimes allowed enough light through in bright conditions to cause "probe off patient" to appear incorrectly on the display, and extra shielding was required in these circumstances. The instrument has an alarm that can be programmed to sound in response to preset $\mathrm{SaO}_{2}$ and pulse limits as well as to a low quality signal. It can be silenced permanently by minor internal modification. The internal battery can power the instrument only for up to two hours, but stored data are retained when the battery becomes exhausted.

STUDY 1: COMPARISON WITH DIRECT ARTERIAL SAMPLING

The comparisons of arterial values and those simultaneously recorded by the three different probes are shown as scatter plots in figure 1 . There is excellent agreement, particularly at higher $\mathrm{SaO}_{2}$ levels, the flex probe proving overall to give values nearest to arterial values. Estimated $\mathrm{SaO}_{2}$ in one chronically hypoxic patient was consistently greater than $\mathrm{SaO}_{2}$ with the oximeter but this discrepancy was corrected when allowance was made for her abnormal $P_{50}(29 \mathrm{~mm} \mathrm{Hg}$ $(3.9 \mathrm{kPa})$ measured by tonometry) in the calculation of $\mathrm{SaO}_{2}$. The mean and standard deviation of the percentage $\mathrm{SaO}_{2}$ differences between individual oximeter and arterial readings (representing bias and precision respectively) were $-0.1(3.4),-0.4(1.8)$, and -0.5 $(2 \cdot 9)$ for ear, flex, and finger probes respectively.

\section{STUDY 2: SPEED OF RESPONSE}

With ear probes, the times taken for the Hewlett Packard, Biox 2A, and Ohmeda 3700 oximeters to detect a rise following sudden onset of oxygen breathing were 9.3 (SD 3.2), 11.1 (2.8), and 9.8 (2.6) seconds. These differences are not significant, but the finger and toe probe times $(23.6(4 \cdot 1), 56 \cdot 8(15 \cdot 8) \mathrm{s}$ respectively) were proportionately longer because of the greater lung to probe site circulation times.

STUDY 3: ASSESSMENT OF ARTEFACT DETECTION Using an arterial cuff above the probe site we were able to show that it was impossible to cause the machine to record a saturation fall of more than $2 \%$ without it registering "low quality signal." If inflation of the arterial cuff is combined with repeated movement of the finger, so simulating a pulse, the instrument may then record a false saturation fall without registering "low quality signal." Unless the movement was similar to the previous heart rate, however, this could usually be detected by inspection of the simultaneous pulse rate signal. 

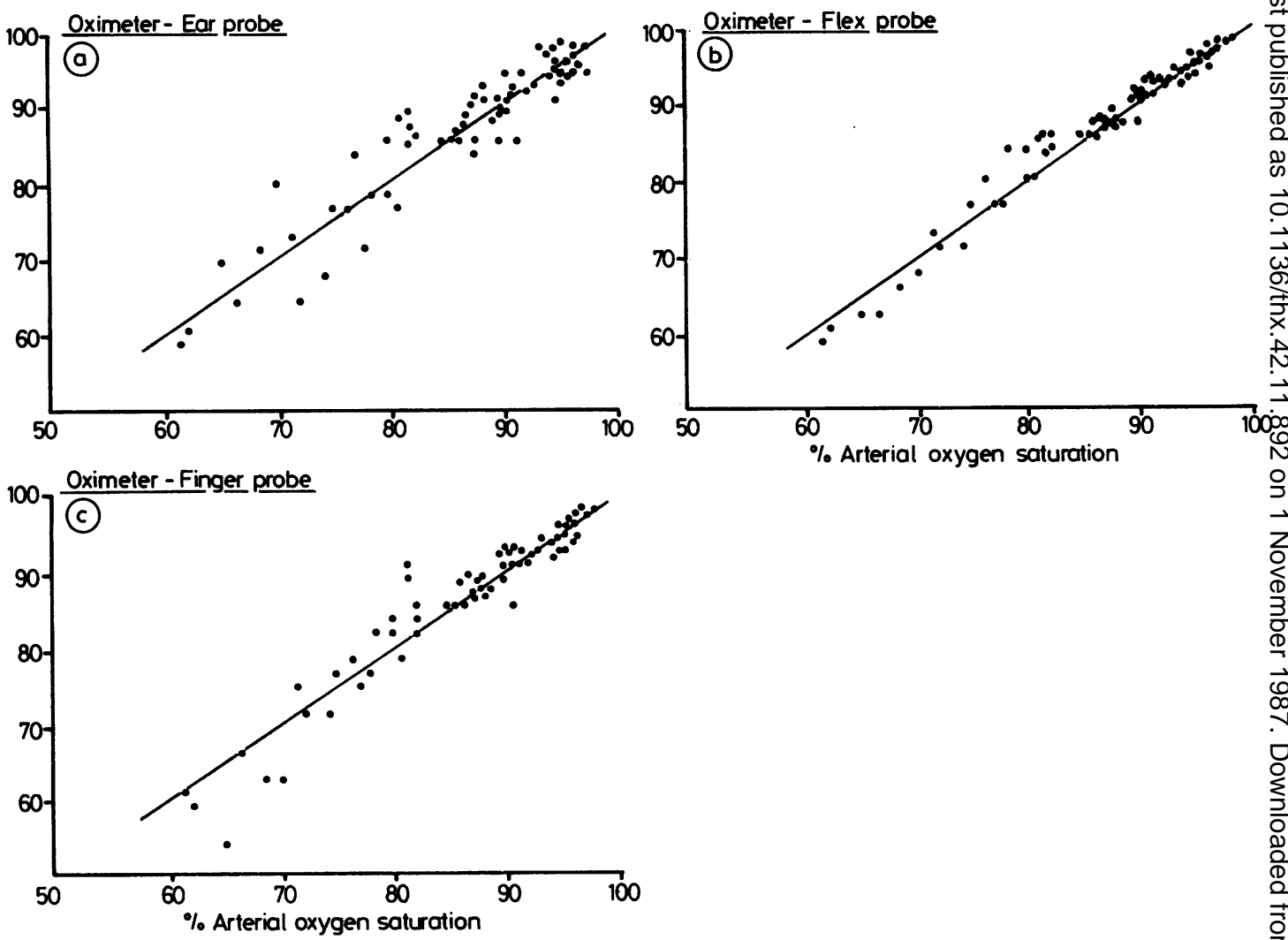

Fig 1 Relationship between the estimated percentage arterial oxygen saturation $\left(\%_{\mathrm{SaO}}\right)$ value from arterial blood gas tensions ( $x$ axis) and the $\% \mathrm{SaO}_{2}$ from the Ohmeda 3700 oximeter (y axis). $a$-ear probe; $b$-flex probe; $c$-finger probe. Th line is the line of identity.

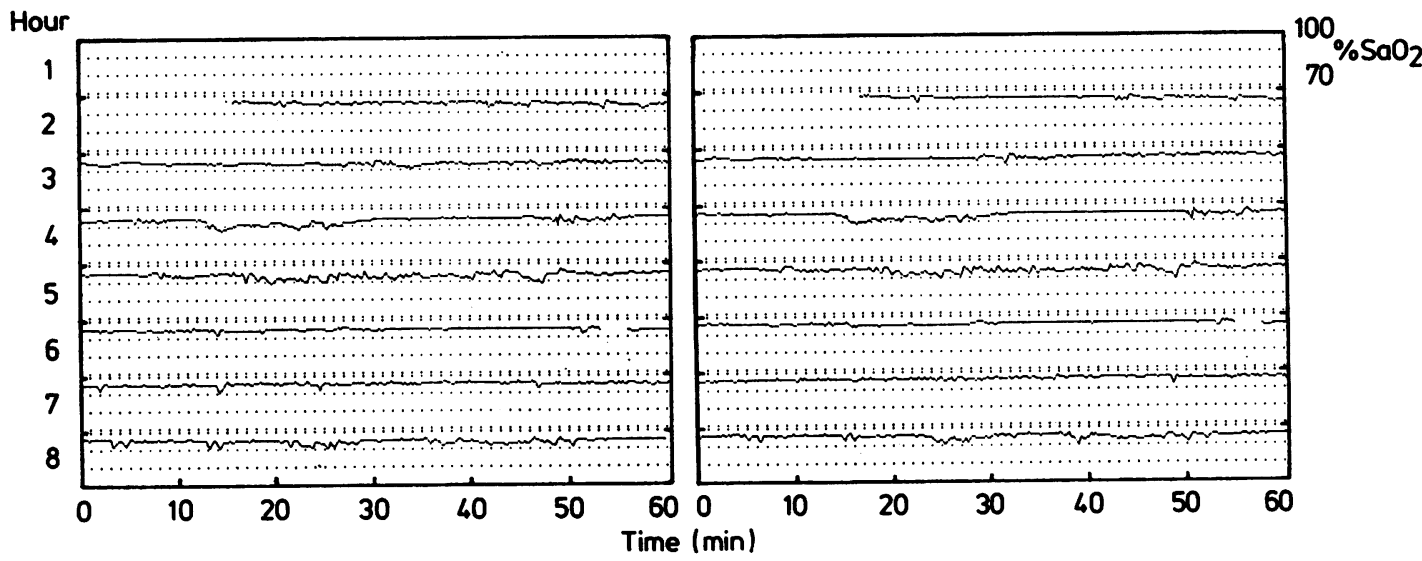

Fig 2 Two overnight oximeter tracings, each from a flexprobe on a finger from opposite hands. Note that any dips usually occur on both tracings. 

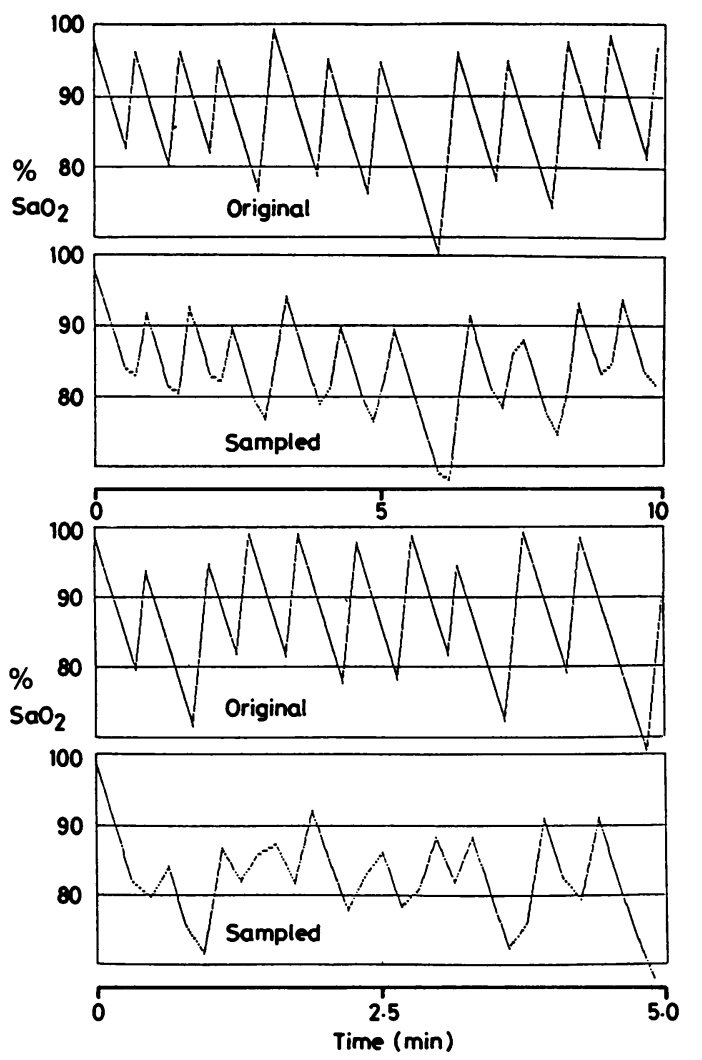

Fig 3 Two computer simulations of fluctuating saturation (top line) and the recreated signal from the memory (bottom line) using the 12 second sampling algorithm (points joined) for two approximate cycle lengths, 60 seconds and 30 seconds.

The five pairs of overnight tracings from opposite hands were very similar (fig 2). There were never more than three $\mathrm{SaO}_{2}$ dips of more than $2 \%$ appearing on one of the paired tracings alone over the eight hour record. The greatest difference in the whole night mean value of $\mathrm{SaO}_{2}$ between a pair of records was $0.9 \%$.

\section{STUDY 4: EFFECT OF SAMPLING ALGORITHM ON} SIGNAL SHAPE

A 12 second sampling frequency on the basis of spot samples should in theory allow reasonable resolution of oscillations with a wavelength down to 30 seconds. This proved to be true for the sampling algorithm used by the oximeter when storing data to memory, with some deterioration when the wavelength was less than 35 seconds. Figure 3 shows phasic dipping in $\mathrm{SaO}_{2}$ at two different approximate frequencies and the recreated pattern from the 12 second sampling algo- rithm. Because the lowest $\mathrm{SaO}_{2}$ observed in the previous 12 seconds was stored rather than a regular point sample, the lowest values are of course the same value as the original; but the maximum recovery $\mathrm{SaO}_{2}$ is underestimated.

\section{Discussion}

The Ohmeda 3700 pulse oximeter (using the flex probe) measured steady state arterial oxygen saturation, with an agreement of $+3.2 \%$ and $-4.0 \% \mathrm{SaO}_{2}$ ( $95 \%$ confidence limits) when compared with values derived from direct arterial sampling. Its mode of action ensures that it can issue a warning when the signal is inadequate for an accurate estimation of $\mathrm{SaO}_{2}$. We have shown its accuracy compared with arterial samples to be at least as good as the previous Biox $2 \mathrm{~A},{ }^{14}$ and with the flex probe possibly a little better. Tweeddale and Douglas ${ }^{3}$ found a mean difference of $1.5 \%$ (SD 3.0\%) using the ear probe and Biox $2 A$, similar to our figures $(-0 \cdot 1 \%(3.4 \%)$ with the ear probe. The response characteristics to changes in $\mathrm{SaO}_{2}$ were similar to the Biox 2A and HewlettPackard 47201A oximeters, and use of the ear probe would allow comparable measurements of hypoxic ventilatory drive by the method of Rebuck and Campbell. ${ }^{6}$

The memory will not store a low quality signal (for example, one due to lying on the probe site) and a blank appears in the data. This ability to reject low quality signals and the eight hours of memory make the Ohmeda 3700 particularly useful for unsupervised screening of oxygen saturation. The 12 second sampling algorithm, saving the lowest $\mathrm{SaO}_{2}$ in that period, allows adequate reproduction of an oscillating signal down to a wavelength of 30 seconds and ensures that the lowest arterial oxygen saturation is not missed. If an inaccurate signal caused by simultaneous arterial compression and probe site movement is read into the memory, this should be detectable by simultaneous inspection of the pulse rate tracing, which will usually display considerably more variation than is usual in the pulse rate, owing to the irregular movement of the probe site that simulated a pulse. The eight hours of memory can be unloaded in eight minutes, and the data subsequently analysed with the help of a microcomputer.

The Ohmeda 3700 pulse oximeter is at present under evaluation in our department as a screening tool for the detection and diagnosis of hypoxaemia during sleep. We have found that patients with obstructive sleep apnoea commonly have a cyclical dipping wavelength in arterial oxygen saturation of 45 to 60 seconds, and the Ohmeda 3700 therefore appears to be technically satisfactory for unattended use in screening for nocturnal hypoxia. 


\section{References}

1 Chapman KR, A D'Urzo, AS Rebuck. The accuracy and response characteristics of a simplified ear oximeter. Chest 1983;83:860-4.

2 Douglas NJ, HM Brash, PK Wraith, et al. Accuracy, sensitivity to carboxyhaemoglobin, and speed of response of the HP-47201A ear oximeter. Am Rev Respir Dis 1979;119:311-3.

3 Tweeddale PM, Douglas NJ. Evaluation of Biox IIA ear oximeter. Thorax 1985;40:825-7.

4 Chapman KR, Liu FLW, Watson RM, Rebuck AS. Range of accuracy of two wavelength oximetry. Chest 1986;89:540-2.

5 Severingaus JW. Blood gas calculator. J Appl Physiol 1966;21:1108-16.

6 Rebuck AS, Campbell EJ. A clinical method for assessing the ventilatory response to hypoxia. Am $\operatorname{Rev} \vec{\circ}$ Respir Dis 1973;109:345-50. 